\section{Case Reports in Ophthalmology}

Case Rep Ophthalmol 2020;11:442-447

DOI: $10.1159 / 000509339$

Published online: August 6, 2020
(C) 2020 The Author(s)

Published by S. Karger AG, Basel www.karger.com/cop

\title{
A Human Amniotic Membrane Plug to Treat Persistent Macular Hole
}

\author{
Luca Ventre Paola Marolo Michele Reibaldi \\ Eye Clinic Section, Department of Surgical Sciences, University of Turin, Turin, Italy
}

\section{Keywords}

Human amniotic membrane $\cdot$ Macular hole $\cdot$ Vitreoretinal surgery

\begin{abstract}
The human amniotic membrane (hAM) has lately demonstrated interesting potential in its retinal applications. Nowadays, the treatment of persistent macular holes is still a challenge. In this paper, we present a case of a hAM plug used to treat a persistent macular hole, with the purpose of highlighting the critical issues of this new surgical procedure. We performed a 23$G$ pars plana vitrectomy and inserted a hAM plug (diameter of $1.5 \mathrm{~mm}$ ) under the neuroretinal margins of the hole (diameter of $657 \mu \mathrm{m}$ ). A complete ophthalmic examination with the assessment of best-corrected visual acuity (logMAR units, ETDRS letter score) and optical coherence tomography (OCT) scans were collected at $1,4,6$, and 10 weeks after surgery. Visual acuity changed from logMAR 1.0 (1) preoperatively to logMAR 1.0 (2), 1.0 (4), 0.9 (9), and 0.9 (10) at $1,4,6$, and 10 weeks of follow-up, respectively. The 1 week postoperative OCT did not show neuroretinal adhesion over the plug, but the following visits showed reattachment of the margins with partial neuroretinal ingrowth. These findings suggest that the correct adaptation of the size of the hAM plug to the macular hole is essential to allow the anatomical closure of the hole and potential functional results.




\section{Case Reports in Ophthalmology}

\section{Introduction}

The human amniotic membrane (hAM) is an easily available tissue which has been already used for many ophthalmic applications. It is composed of an avascular stromal matrix and a basement membrane with a single layer of epithelium. Besides all its well-known applications in anterior segment surgery, recent preclinical studies demonstrated that the amniotic membrane (AM) is a valid support matrix for retinal pigmented epithelium (RPE) restoration $[1,2]$. Ohno-Matsui et al. [1] proved that human RPE cells cultured on the basement membrane side of dispase-treated AM did not lose their epithelial phenotype morphologically and showed an upregulation of many growth factors. The time required for RPE cells to regenerate was within $24 \mathrm{~h}$ of seeding. Furthermore, hAM had good results on RPE cells in the subretinal space in pigs [3]. In vivo hAM plug transplantation was recently adopted in a case of choroidal hole repair in a globe rupture [4] and in some cases of retinal breaks associated with retinal detachments and recurrent macular hole treatment [5-7]. Currently, the treatment of persistent macular holes is still a challenge. The failure rate of primary surgery in idiopathic macular holes is less than $10 \%$ [8]. Possible causes are residual epiretinal traction, insufficient gas tamponade, poor compliance by the patient in keeping face-down position, or unknown. After primary vitrectomy with peeling of the internal limiting membrane (ILM), it is difficult to find anatomic alternatives to be used as a plug inside the macular hole. Lately, modern approaches aimed to achieve the following goal: the use of an ILM plug peeled from the periphery of the posterior pole [9], a neurosensory retinal free flap [10, 11], or capsular lens fragments [12, 13]. These techniques are difficult to handle and have a high rate of serious complications, such as intraoperative hemorrhages and retinal detachments.

Here, we report the case of a patient with persistent macular hole who underwent hAM implantation surgery. Our goal is to highlight the critical aspects of this new technique and to show which of them can impact on the anatomical and functional final outcomes.

\section{Case Report}

We present the case of a 70-year-old female patient who had undergone a pars plana vitrectomy performed with peeling of the ILM and gas tamponade for an idiopathic macular hole. She was referred to our clinic 6 months later and diagnosed with a large persistent macular hole, which remained open despite the previous surgery.

Preoperatively and at every follow-up visit $(1,4,6$, and 10 weeks), a complete ophthalmic examination was performed. It included refraction with the assessment of best-corrected visual acuity (logMAR units, ETDRS letter score), Goldmann applanation tonometry, standard fundus-dilated ophthalmic examination and spectral domain optical coherence tomography (OCT) (Spectralis HRA+OCT; Heidelberg Engineering, Heidelberg, Germany) with a manual measurement of the internal diameter of the macular hole with the inbuilt caliper tool. Every examination was taken by the same examiner, and the follow-up setting of the machine was used to perform OCT over time.

The hAM was stored and cryopreserved in the tissue bank, located in our center in Torino. It was defrosted when delivered to our operating room. The hAM was cut intra-operatively with a full-thickness biopsy punch of $1.5 \mathrm{~mm}$. It was then inserted in the vitreous chamber and in the subretinal space corresponding to the macular hole.

The surgical procedure was carried out under retrobulbar anesthesia of lidocaine and ropivacaine mixed in equal volumes. A standard 3-port 23-G transconjunctival pars plana 


\section{Case Reports in Ophthalmology}

vitrectomy (Optikon $2000 \mathrm{SpA}$, Rome, Italy) was performed. A hAM plug of $1.5 \mathrm{~mm}$ (diameter of the punch) was cut from its patch and then placed under the neuroretinal margins of the hole, which was $657 \mu \mathrm{m}$ in diameter at its wider part (Fig. 1).

The plug was inserted inside the vitreous cavity through a $23-\mathrm{G}$ trocar after valve removal. The hAM was positioned through the macular hole into the subretinal space, with its basal membrane faced up, and the chorion layer faced towards the RPE. The edges of the hole were stretched and raised to allow insertion of the plug into a hole with a smaller diameter. Inserting the hAM above the RPE, the margins of the hole where carefully detached and the hAM inserted under them, but the hAM still presented some folds at the end of the surgery.

A fluid-air exchange was performed at the end of the surgery and the sclerostomies were sutured. Afterward, an injection of $0.8 \mu \mathrm{m}$ of pure sulfur hexafluoride (SF6) was chosen as temporary endotamponade. Face-down positioning was recommended to the patient for 3 days after surgery.

Preoperative visual acuity (logMAR, ETDRS letter score) was 1.0 (1). The 1-week postoperative OCT did not show neuroretina adhesion over the hAM plug (Fig. 2). Visual acuity was $\operatorname{logMAR} 1.0$ (2). The 4-week postoperative OCT showed reattachment of the margins and partial neuroretinal ingrowth over the hAM plug, which was greater at the 6- and 10-week postoperative OCT (Fig. 2), with no significant change of visual acuity: logMAR 1.0 (6) at 4 weeks, logMAR 0.9 (9) at 6 weeks, and logMAR 0.9 (10) at 10 weeks. No adverse events such as endoocular inflammation or an increase of intraocular pressure were recorded during the followup period.

\section{Discussion/Conclusion}

We performed a new surgical technique, recently proposed by Rizzo and colleagues [5-

7], which provides the transplantation of a hAM plug into the subretinal space in recurrent macular holes. This approach is indicated in eyes with persistent macular holes which have already undergone pars plana vitrectomy with peeling of the ILM.

hAM patches are easy to obtain from the tissue bank and are also easy to manipulate inside the eye, differently from other techniques recently proposed, such as an autologous ILM plug [9], neurosensory retinal free flap $[10,11]$, or capsular lens fragments $[12,13]$. The other techniques cannot promote retinal function restoration and surgical techniques require a longer surgical time and are more difficult, while the hAM plug has a potential role in the promotion of neurosensory retinal regeneration. However, according to our results, further studies are needed to demonstrate its regenerative potential. The presence of growth factors secreted by the AM epithelial cells can stimulate neuroepithelial regeneration. Furthermore, studies on animals [14] showed that AM cells promote the recovery of neurologic functions. No immunogenic issues have been considered, as the AM is already widely used in ophthalmic anterior segment surgery and has no immunogenicity problems.

In our case, a 10-week follow-up OCT showed partial regrowth of the retinal layers above the hAM. At 1 week the margins of the macular hole were still open. In contrast, Rizzo et al. [5] demonstrated complete macular hole closure with the neurosensory retina totally overfilling the hAM plug in all cases of hAM macular hole transplantation after 1 week (when the gas tamponade disappeared); therefore our result was not as we expected. We had two choices: inject an air bubble inside the vitreous chamber or watch and wait. We decided on the second option and registered the development of the hole. We noted the advance of the neuroretina above the hAM over time; we found that the hole was progressively closing at OCT imaging 


\section{Case Reports in Ophthalmology}

Ventre et al.: A Human Amniotic Membrane Plug to Treat Persistent Macular Hole

from 1 week to 10 weeks postoperatively. The hAM was contracting, dragging the margins of the hole with it. Besides the partial closure of the hole, we could detect a very small visual change from $1.0 \log$ MAR (1) to $0.9 \log$ MAR (10) according to the ETDRS letter score.

Considering our findings, we suggest two possible interpretations. The first is a regenerative effect of the hAM on the neurosensory retina, with its margins progressively moving forward over the membrane. The second is that the progressive closure of the hole could simply be due to the shrinkage of the hAM with the retina in tow over time (see the hAM measurements in Fig. 2).

Regarding the persistence of the detachment of the margin 1 week after surgery, we think that the cause is the different dimension between the plug and the hole (punch of $1.5 \mathrm{~mm}$, hole of $650 \mu \mathrm{m}$ ), leading to a centrifugal thrust against the edges of the hole. According to our experience, we suggest paying particular attention to the correct calibration of the membrane dimensions with respect to the hole, and we also consider that a minimal oversizing of the hAM could lead to a delay in the hole closure due to its implied mechanical obstacles.

\section{Statement of Ethics}

In accordance with the tenets set forth in the Declaration of Helsinki, written informed consent to publish this case was obtained from the patient.

\section{Conflict of Interest Statement}

The authors have no conflicts of interest to declare.

\section{Funding Sources}

The authors received no financial support for the research, authorship, and/or publication of this article.

\section{Author Contributions}

L.V., P.M. and M.R.: equal contribution to patient management, conception of the paper, data analysis and interpretation, manuscript drafting, and literature search. All authors read and approved the final manuscript.

\section{References}

1 Ohno-Matsui K, Ichinose S, Nakahama K, Yoshida T, Kojima A, Mochizuki M, et al. The effects of amniotic membrane on retinal pigment epithelial cell differentiation. Mol Vis. 2005 Jan;11:1-10.

2 Capeáns C, Piñeiro A, Pardo M, Sueiro-López C, Blanco MJ, Domínguez F, et al. Amniotic membrane as support for human retinal pigment epithelium (RPE) cell growth. Acta Ophthalmol Scand. 2003 Jun;81(3):271-7.

3 Kiilgaard JF, Scherfig E, Prause JU, la Cour M. Transplantation of amniotic membrane to the subretinal space in pigs. Stem Cells Int. 2012;2012:716968.

4 Zhu D, Jin X, Zhou J. Transplantation of amniotic membrane for choroidal hole to treat suprachoroidal silicone oil migration. Acta Ophthalmol. 2017 Sep;95(6):e522-3. 


\section{Case Reports in Ophthalmology}

5 Rizzo S, Caporossi T, Tartaro R, Finocchio L, Franco F, Barca F, et al. A human amniotic membrane plug to promote retinal breaks repair and recurrent macular hole closure. Retina. 2019 Oct;39 Suppl 1:S95-103.

6 Caporossi T, Tartaro R, De Angelis L, Pacini B, Rizzo S. A human amniotic membrane plug to repair retinal detachment associated with large macular tear. Acta Ophthalmol. 2019 Dec;97(8):821-3.

7 Caporossi T, De Angelis L, Pacini B, Tartaro R, Finocchio L, Barca F, et al. A human amniotic membrane plug to manage high myopic macular hole associated with retinal detachment. Acta Ophthalmol. 2020 Mar;98(2):e252-e256. https://doi.org/10.1111/aos.14109.

8 Zhao P-P, Wang S, Liu N, Shu Z-M, Zhao J-S. A review of surgical outcomes and advances for macular holes. J Ophthalmol. 2018 Apr;2018:7389412.

9 Morizane Y, Shiraga F, Kimura S, Hosokawa M, Shiode Y, Kawata T, et al. Autologous transplantation of the internal limiting membrane for refractory macular holes. Am J Ophthalmol. 2014 Apr;157(4):861-869.e1.

10 Grewal DS, Mahmoud TH. Autologous neurosensory retinal free flap for closure of refractory myopic macular holes. JAMA Ophthalmol. 2016 Feb;134(2):229-30.

11 Thomas AS, Mahmoud TH. Subretinal transplantation of an autologous retinal free flap for chronic retinal detachment with proliferative vitreoretinopathy with and without macular hole. Retina. 2018 Sep;38 Suppl 1:S121-4.

12 Chen SN, Yang CM. Lens capsular flap transplantation in the management of refractory macular hole from multiple etiologies. Retina. 2016 Jan;36(1):163-70.

13 Peng J, Chen C, Jin H, Zhang H, Zhao P. Autologous lens capsular flap transplantation combined with autologous blood application in the management of refractory macular hole. Retina. 2018 Nov;38(11):217783.

14 Evans MA, Broughton BR, Drummond GR, Ma H, Phan TG, Wallace EM, et al. Amnion epithelial cells - a novel therapy for ischemic stroke? Neural Regen Res. 2018 Aug;13(8):1346-9.
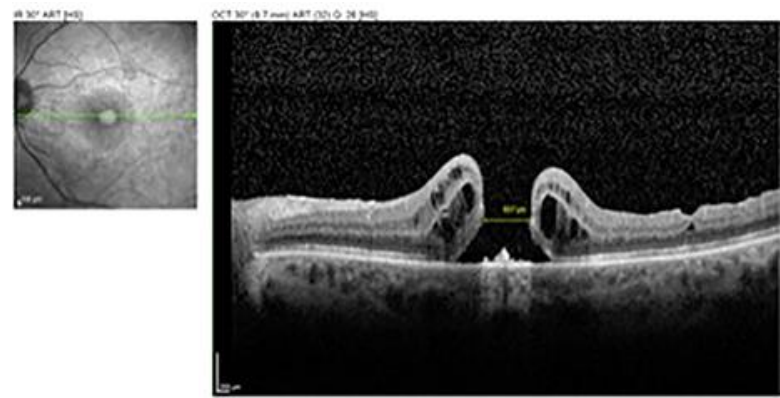

Fig. 1. Preoperative OCT. 
Case Reports in Ophthalmology
Case Rep Ophthalmol 2020;11:442-447

DOI: $10.1159 / 000509339$

(c) 2020 The Author(s). Published by S. Karger AG, Basel www.karger.com/cop

Ventre et al.: A Human Amniotic Membrane Plug to Treat Persistent Macular Hole
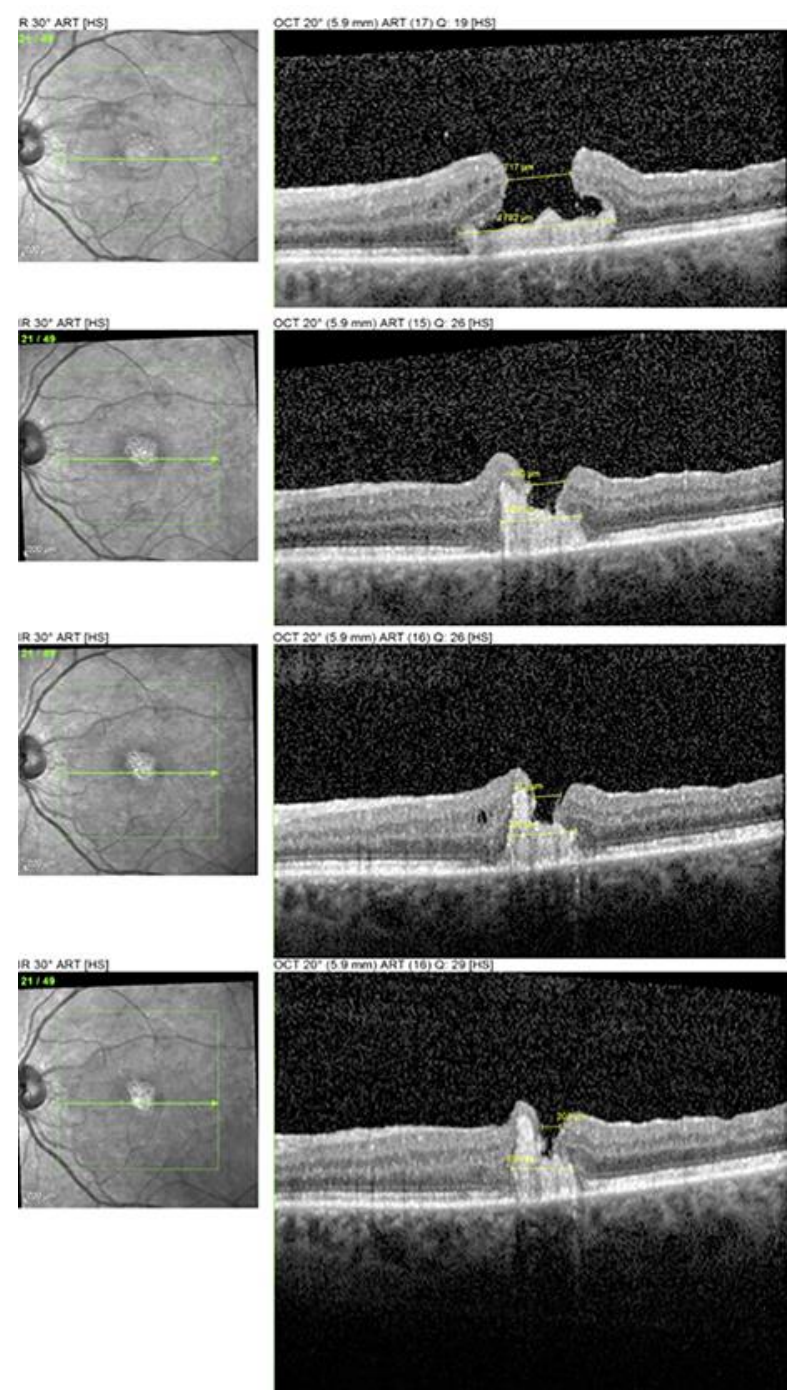

Fig. 2. Follow-up OCT scans at 1, 4, 6, and 10 weeks after hAM implant. 